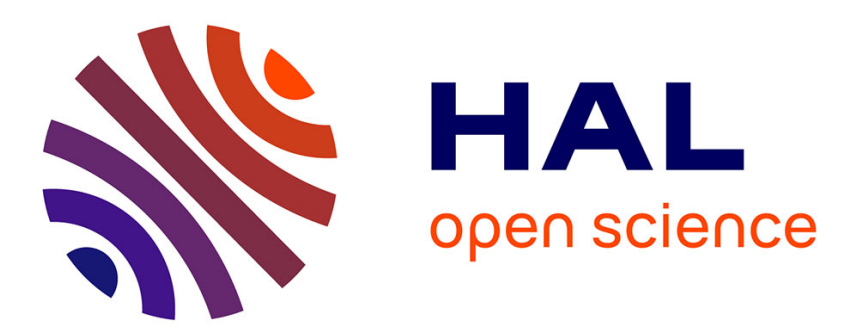

\title{
Three-dimensional holographic photostimulation of the dendritic arbor
}

\author{
Sunggu Yang, Eirini Papagiakoumou, Marc Guillon, Vincent de Sars, \\ Cha-Min Tang, Valentina Emiliani
}

\section{> To cite this version:}

Sunggu Yang, Eirini Papagiakoumou, Marc Guillon, Vincent de Sars, Cha-Min Tang, et al.. Threedimensional holographic photostimulation of the dendritic arbor. Journal of Neural Engineering, 2011, 8 (4), pp.046002. hal-01976677

\section{HAL Id: hal-01976677 https://hal.science/hal-01976677}

Submitted on 10 Jan 2019

HAL is a multi-disciplinary open access archive for the deposit and dissemination of scientific research documents, whether they are published or not. The documents may come from teaching and research institutions in France or abroad, or from public or private research centers.
L'archive ouverte pluridisciplinaire HAL, est destinée au dépôt et à la diffusion de documents scientifiques de niveau recherche, publiés ou non, émanant des établissements d'enseignement et de recherche français ou étrangers, des laboratoires publics ou privés. 


\title{
3D Holographic Photostimulation of the Dendritic Arbor
}

\section{Sunggu Yang ${ }^{1}$, Eirini Papagiakoumou², Marc Guillon², Vincent de Sars², Cha-Min Tang ${ }^{1}$, and Valentina Emiliani ${ }^{2 \S}$}

\author{
${ }^{1}$ Department of Neurology, University of Maryland School of Medicine, 655 West Baltimore St, \\ and Baltimore VAMC, 10 N. Greene St, Baltimore, Maryland 21201 USA \\ 2 Neurophysiology and New Microscopies Laboratory, Wave front engineering microscopy \\ group, CNRS UMR 8154, INSERM U603, Paris Descartes University, 45 Rue des Saints Pères, \\ 75270 Paris Cedex 06, France \\ $\S$ Corresponding author: valentina.emiliani@parisdescartes.fr
}

\begin{abstract}
:
Digital holography is an emerging technology that can generate complex light patterns for controlling the excitability of neurons and neural circuits. Strengths of this technique include the high efficiency with which available light can be effectively utilized and the ability to deliver highly focused light to multiple locations simultaneously. Here we demonstrate another power of digital holography, the ability to generate instantaneous three dimensional light patterns. This capability is demonstrated with photolysis of caged glutamate on the dendritic arbor of hippocampal neurons to study the nature of integration of inputs arriving on multiple dendritic branches.
\end{abstract}

\section{Introduction:}

Light can be a powerful medium with which the excitability of the nervous system can be probed [1]. When used in conjunction with caged compounds or in optogenetically engineered tissue, light can provide the means to control the strength, timing, and location of stimulation with unparalleled precision. Such control can be achieved without direct contact of the tissue and with little signal crosstalk. 
An ideal light-delivering method should be one that is efficient (minimal power loss throughout the system), flexible (capable of targeting a wide range of samples, from a single dendritic spine to a whole 3D population of neurons), and has high resolution in time and space (millisecond and micrometric, respectively). Several solutions have been recently proposed to fulfill these requirements. They can be divided in two main categories: laser scanning and parallel excitation methods. Each of these approaches has advantages and limitations.

Laser scanning methods use galvanometric mirrors or acousto-optic devices (AOD) to quickly scan a laser beam across several positions [2-5]. One limitation of the laser scanning approach is the time it takes to scan through a sequence of locations when the geometry is complex and when it involves a large number of spots.

This can be especially problematic for photolysis of caged compounds because a finite dwell time is required at each spot regardless of the available power of the light source. The dwell time is a consequence of inefficient photolysis of available caged compounds [4], particularly in twophoton [6,7], depletion of available caged compounds from a very small volume, and light intensity limitation set by the photodamage threshold of the tissue [8]. The dwell time problem is accentuated when there is a need to stimulate a large number of synapses. Under a wide range of physiological conditions large numbers of synapses on a single neuron must be activated within a brief temporal window to generate an action potential. If the inputs are asynchronous they are then typically summed linearly or sub-linearly even when spatially clustered, while supra-linear summation occurs only in the case of near simultaneous arrival of stimuli [9]. Temporal coincidence of paired excitation is also critical for the induction of synaptic and dendritic plasticity [10]. Therefore, a method that can provide excitation at a large numbers of sites simultaneously will be helpful for studying plasticity as well as dendritic integration.

With parallel methods all the regions in the target area are excited simultaneously. They are based either on the modulation of light intensity (via digital micromirror devices (DMD), liquid crystal (LC) and light-emitting diode (LED) arrays [11]) or on the modulation of the phase of a coherent beam (via nematic or ferroelectric liquid crystal arrays). DMD is an example of amplitude modulating device [12-16]. A DMD is a semi-conductor chip with hundreds of thousands of switchable micromirrors that can be digitally controlled to produce complex two dimensional patterns. Its strength includes tolerance to high light intensity and compatibility with a wide range of wavelength, as well as switching times in the tens of microseconds range. Nematic- or ferroelectric-LC allows controlling light distribution at the focal plane by a pure 
phase modulation following the notion of image reconstruction in holography. Originally proposed for generating multiple traps in optical tweezers [17-21], the experimental scheme for holographic light patterning consists in computing with an iterative algorithm the phase pattern at the rear aperture of the objective that reproduces the desired target intensity in the objective focal plane.

With amplitude modulation, light patterns are created by selectively blocking light, while with phase modulation light patterns are creating by "redirecting" the light onto the target [22]. The result is a marked difference in light utilization efficiency when generating sparsely distributed light patterns.

Another significant difference between these two technologies is the ability to generate 3D patterns. In biology the structures of interest are normally three dimensional. But current imaging and optical stimulation approaches are fundamentally two dimensional technologies. While they can be modified to handle 3D structures, this is generally achieved at a cost of speed and efficiency [23]. Biologists have responded to this application-technology mismatch by either forcing the tissue into a $2 \mathrm{D}$ state (i.e. culturing it on a glass coverslip) or placing constraints on the parameter that is being measured. For example, the dendritic arbor of a neuron is in general a complex 3D structure so that dendritic integration with $2 \mathrm{D}$ approaches requires confining the excitation to relatively short portion of a dendrite which lies in the imaging plane of the microscope. This constraint makes it difficult to address important questions such as the nature of multi-branch integration and integration over an extended length of a single dendrite.

On the contrary, holographic light patterning is inherently a 3D technology. Holographic phase modulation through liquid crystal spatial light modulators (LC-SLM) was originally proposed for generation of 3D optical traps [17-21]. Its use for 3D imaging or photoactivation has been recently proposed and discussed [24-26] and the generation of 3D focused spots has been demonstrated by exciting fluorescent test samples [26, 27]. However, a demonstration for 3D photoactivation in a biological preparation has not yet been reported. Here, we present and characterize a holographic microscope system for 3D photolysis and demonstrate applications in experimental neuroscience with photolysis of caged glutamate on the complex 3D architecture of the dendritic neurons.

\section{3D holographic spot generation}

\subsection{Experimental set up}


Three dimensional control of multiple spot generation is achieved by using a conventional holographic configuration [24, 28]. Briefly, the output beam of a $150 \mathrm{~mW}, 405 \mathrm{~nm}$ diode laser (CNI Laser) is expanded by a beam expander (3X) to fill the short axis of a reflective Spatial Light Modulator (SLM) (LCOS Hamamatsu, model X10468-05). The SLM plane is imaged at the back aperture of the microscope objective through a telescope $(\mathrm{L} 1, \mathrm{fl}=750 \mathrm{~mm}$; L2, $\mathrm{f} 2=500 \mathrm{~mm}$ ). The magnification of the telescope is chosen in order to match the SLM short axis with the diameter of the objective's back aperture (Olympus, 60x, W 0.9NA). The undiffracted component (zero order spot) is removed by placing an aluminum block at the focal plane of L1. For the photolysis experiments, the holographic illumination system was combined with electrophysiological recording and conventional fluorescence imaging. Temporal control of the light pulse was accomplished by gating the laser.

The holographic beam was brought into the optical axis of an upright fluorescence microscope (Olympus BX51) below the epi-fluorescence unit, with a longpass dichroic mirror. The intracellular solution of the whole cell patch clamp electrode contained Alexa594 $(50 \mu \mathrm{M})$ or Cascade Blue hydrazide $(750 \mu \mathrm{M}))$ to identify the location of the thin terminal dendrites and dendritic spines. Internal solution consisted of (in $\mathrm{mM}$ ): $135.0 \mathrm{~K}$-gluconate, $5 \mathrm{KCl}, 1 \mathrm{MgCl}_{2}$, $0.02 \mathrm{CaCl}_{2}, 0.2$ EGTA, 10 HEPES, $4 \mathrm{Na}_{2}$-ATP, and 0.3 Na-GTP. External solution consisted of (in $\mathrm{mM}$ ): $124 \mathrm{NaCl}, 4 \mathrm{KCl}, 1.23 \mathrm{NaH}_{2} \mathrm{PO}_{4}, 1.5 \mathrm{MgCl}_{2}, 2.5 \mathrm{CaCl}_{2}, 26 \mathrm{NaHCO}_{3}$ and 10 glucose. Caged glutamate (MNI-Glu, 1-2 mM) was bath applied and recirculated at a rate of $0.7 \mathrm{ml} / \mathrm{min}$. Experiments were done on hippocampal acute slices, at room temperature. Data were acquired with pClamp10 software.

\subsection{Phase hologram calculation}

To locate the excitation spot on the sample plane and calculate the phase holograms, we used a custom designed software written in C++ with the open graphic library Qt 4.4.0 [24].

In order to minimize the effects of spherical aberrations on the spots generated out of the focal plane, for the calculation of phase profiles we included in the description of the microscope objective the non parabolic terms. Briefly, for a high numerical aperture lens satisfying quasistigmatism sine condition, a ray coming from a source placed at the focus and forming an angle $\alpha$ with the optical axis, will emerge from the lens back aperture at distance $\mathrm{d}=f \cdot \sin (\alpha)$ with a

phase $\Delta \varphi=\frac{2 \pi . m}{\lambda} f, m$ is the index of refraction of the embedding medium and $f$ is the focal 
length of the lens in the medium, and $\lambda$ the optical wavelength. Any ray coming from the focus will leave the lens with the same phase, yielding a plane wave. The lens thus, flattens and projects the incoming spherical wave of radius $f$ on the back aperture. If we, now, consider (figure 1 (a)) a ray coming from a point source $\mathrm{N}$ (coordinates $\mathrm{x}_{\mathrm{n}}, \mathrm{y}_{\mathrm{n}}$, and $\mathrm{z}_{\mathrm{n}}$ ) placed out of focus in the sample volume, and reaching the "focal sphere" [29] at a point $P$ (coordinates $x_{p}, y_{p}, z_{p}$ with $\left.z_{p}=\sqrt{f^{2}-\mathrm{x}_{\mathrm{p}}{ }^{2}-\mathrm{y}_{\mathrm{p}}{ }^{2}}\right)$, we can write its phase at the focal sphere, and thus at the lens back aperture, as $\Delta \varphi_{p}^{n}=\frac{2 \pi \cdot m}{\lambda} N P=\frac{2 \pi \cdot m}{\lambda} \sqrt{\left(x_{p}-x_{n}\right)^{2}+\left(y_{p}-y_{n}\right)^{2}+\left(z_{p}-z_{n}\right)^{2}}$.

Inversely, back propagation of a ray having the former phase at the back aperture of the objective will give a spot at point $N$. Let's then define $u_{p}=|u| \exp \left(i \phi_{p}\right)$ the complex electric field at the $p$ th pixel of the SLM, imaged in the back aperture of the objective, and use the scalar diffraction theory to propagate this field distribution, through an objective of focal $f$, to the location of the $n$th spot at the sample space. The complex amplitude $v_{n}$ at the $n$th spot can be found as the sum of the $\mathrm{N}$ contributions from all the pixels of the SLM:

$$
v_{n}=\frac{d^{2}}{i \lambda f} \sum_{p=1, N} \mid u e^{i\left(\phi_{p}-\Delta \phi_{p}^{n}\right)} \text {, for small defocus compared to the objective focal length }\left(\mathrm{z}_{\mathrm{n}} \ll f\right) \text { the }
$$

expression of $\Delta \varphi_{\mathrm{p}}^{\mathrm{n}}$ can be simplified to: $\Delta \varphi_{p}^{n}=\frac{2 \pi \cdot m}{\lambda f}\left(1+x_{n} x_{p}+y_{n} y_{p}+z_{n} \sqrt{f^{2}-x_{p}^{2}-y_{p}^{2}}\right)$

which for low numerical aperture objectives,,$\left(\mathrm{x}_{\mathrm{p}}^{2}+\mathrm{y}_{\mathrm{p}}^{2}<<f\right)$ can be further simplified in: $\Delta \varphi_{p}^{n}=\frac{2 \pi \cdot m}{\lambda f}+\frac{2 \pi \cdot m}{\lambda f}\left(x_{n} x_{p}+y_{n} y_{p}\right)-\frac{\pi \cdot m}{\lambda f^{2}} z_{n}\left(x_{p}^{2}+y_{p}^{2}\right)(2) ;$ the last expression, currently used in the prisms and lenses algorithm [30], permits the generation of good optical spots, but generates spherical aberrations for excitation spots out of the objective focal plane with consequent deterioration in intensity and axial resolution. It was shown that the approximation (2) induced significant point spread function degradation above $10 \mu \mathrm{m}$ while modeling high numerical aperture lenses by equation (1) allowed defocusing of $40 \mu \mathrm{m}$ or larger [31]. In order to optimize these parameters, in the calculation of $v_{n}$ we therefore used the expression (1), which hardly increased computation time.

Finally, to optimize the uniformity of light intensity in multiple spots configuration, we used the weighted Gerchberg-Saxton algorithm (GSW) [32]. This multi-iterative algorithm consists in 
attributing a correction weight to the target spots in order to balance numerically-generated inhomogeneities.

\subsection{Optical characterization}

In order to characterize the quality of holographic spots organized in 3D structures, we implemented the holographic scheme, described in section 2.1, on a double microscope where the upper objective (60x, W 0.9 NA) is used for holographic excitation and the lower one for imaging (60x, W 1.2NA) [24]. The imaging objective is fixed and focused on a thin fluorescent layer while the upper one is moved along the axial direction with a piezo scanning stage working in closed-loop (MIPOS100SG, Piezosystem Jena).

An example of a 3D holographic pattern of diffraction-limited spots arranged in a cubic structure is shown in figure 1(b). The pattern is shown from two perspectives (side and top views) to better illustrate the light distribution at the different spot along the axial and transversal plane, respectively.

In order to define the excitation volume available for photolysis experiments, we characterized the full width at half maximum (FWHM) and intensity distribution of the holographic spots as a function of their lateral, $d x$, and axial, $d z$, position. To characterize these parameters for the laser excitation wavelength, $405 \mathrm{~nm}$, we replaced the fluorescent layer with a transparent coverslip and directly imaged the holographic spots through the lower objective into the imaging camera.

A series of spot of coordinates $\mathrm{x}=0, \mathrm{y}=5 \mu \mathrm{m}$ and variable values for the defocus, $d z$, or of coordinates $\mathrm{y}=5, \mathrm{z}=0 \mu \mathrm{m}$ and variable values for the lateral position $d x$ where generated (the lateral shift of $5 \mu \mathrm{m}$ allows avoiding the zero order spot).

The corresponding values for the peak intensity as a function of the lateral and axial displacement are reported in figure $\mathbf{1}(\mathbf{c}-\mathbf{d})$.

In diffractive optics, the lateral intensity distribution is determined by the spatially varying diffraction efficiency, $\delta(d x, d y)$, defined as the ratio between the integrated intensity, $\mathrm{I}_{\text {spot, }}$ redirected into the desired target spot(s) and the integrated light intensity, $\mathrm{I}_{0}$, of the zero order spot (SLM off), i.e. $\delta=\mathrm{I}_{\text {spot }} / \mathrm{I}_{0}$. Its value decreases with increasing distance, $d x$ or $d y$, following in a good approximation the low numerical aperture approximation (red curve in figure 1 (c)):

$$
\delta(d x, d y)=\left(\frac{\sin \mathrm{X}}{\mathrm{X}}\right)^{2}\left(\frac{\sin \mathrm{Y}}{\mathrm{Y}}\right)^{2}
$$


where $X=\left(\frac{m \pi a}{\lambda f} d x\right), Y=\left(\frac{m \pi a}{\lambda f} d y\right), m$ is the refractive index at the sample plane, $f$ is the objective focal plane in water and $a$ is the de-magnified SLM pixel size at the objective's back aperture, i.e. $a=\mathrm{d}_{\mathrm{SLM}} \mathrm{f}_{2} / \mathrm{f}_{1}$ ( $\mathrm{d}_{\mathrm{SLM}}$ being the original pixel size which is $20 \mu \mathrm{m}$ for the model used in this paper). This expression also sets the qualitative limits for the maximum excitation field: $2 \times d x_{\max }=2 \times d y_{\max }=2 \times\left(\frac{\lambda f_{1}}{m \cdot d_{S L M}} \frac{f}{f_{2}}\right)[25,28]$.

Diffraction efficiency also affects light distribution in the longitudinal direction [25]. The loss of energy in this case is due to rays coming from pixels imaged at the edge of the pupil. As pixels diffract light in a limited cone with a half angle of the order of $\lambda / 2 a$, where $a$ is the pixel size, pixels located at the edge of the pupil will diffract on a limited region of the optical axis (figure 1(a)), the range of accessible defocus being of the order of $2 \times d z_{\max } \cong 2 \times \frac{m \lambda f}{2 N A \cdot a}$, where NA is the numerical aperture of the objective. The more this limit is approached the less the pixels at the edge contribute to the total intensity at the spot (the corresponding defect of energy is sent to higher diffraction orders). For the parameters used in our system $2 \times d x_{\max }=2 \times d y_{\max } \approx 180 \mu m$ and $2 \times d z_{\max } \approx 185 \mu m$.

To evaluate this effect in our system, we generated a spot of coordinates $x=0, y=5 \mu m$ and variable values for the defocus, $d z$, and measured experimentally the corresponding integrated intensity. The results are reported in figure 1(d) where the green curve is a numerical simulation. This has been achieved by summing the diffraction patterns from all pixels at the focal sphere. To take into account that the projection of the pixel at the pupil plane onto the focal sphere gives rise to a lateral stretch of the original pixel size, $a$, along $\mathrm{x}$ and $\mathrm{y}$ directions, the pixel at the focal sphere has been represented as a rectangle of sides $a_{x}$, $a_{y}$. This correction permits a very good fitting of the experimental data.

A second consequence of the reduced contribution of the side pixels with increasing defocus is a reduction of the effective numerical aperture and therefore a deterioration of the axial FWHM. To evaluate this effect in our system, we performed for different values of defocus $\mathrm{d} z$ an axial scan of $\pm 5 \mu \mathrm{m}$. By plotting intensity profiles along $\mathrm{x}, \mathrm{y}$ direction we determined the lateral FWHMs by Gaussian fittings. The axial FWHM was measured by fitting the axial intensity by a Lorentzian provided the diffraction efficiency can be considered as constant on such a small $\mathrm{dz}$ 
range. We obtained experimentally that axial FWHMs varies from $1.2 \mu \mathrm{m}$ to $\sim 2 \mu \mathrm{m}$ going from $\mathrm{z}=0$ to $\mathrm{z}=50 \mu \mathrm{m}$ and that lateral FWHMs varies from $0.3 \mu \mathrm{m}$ to $\sim 0.4 \mu \mathrm{m}$ in the same $\mathrm{z}$-range.

These results show that, with the used optical parameters, light efficiency $\geq 50 \%$ and an almost constant lateral and axial FWHMs can be obtained for spot generated in an excitation volume of $80 \times 80 \times 100 \mu \mathrm{m}^{3}$

\section{Holographic Photostimulation of the Dendritic Arbor}

\subsection{Targeting individual dendritic spines}

First we wanted to test the resolution and achievable excitation power for photolysis in a scattering medium and under typical electrophysiological experimental conditions.

To this end, we tested the performance of the system for photostimulation of individual dendritic spines.

A CA1 hippocampal pyramidal neuron was dialyzed through the patch electrode with Cascade Blue hydrazide $(750 \mu \mathrm{M})$, a fluorescent dye that can be excited by the $405 \mathrm{~nm}$ laser beam. Figure 2(a) (left panel) shows the wide-field fluorescence image of a dendritic spine on a dendritic branch $\sim 40 \mu \mathrm{m}$ below the surface. A holographic spot was generated at the position of the selected spine and the corresponding fluorescence image was acquired (middle panel). The two images are then superimposed (right panel). The holographically generated spot has a diameter of $\sim 0.6 \mathrm{~nm}(\mathrm{FWHM})$, which is still close to the value measured at $405 \mathrm{~nm}$ in a non-scattering sample (section 2).

To evaluate the ability of the system to generate the necessary light intensity for photolysis a brief light pulse $(0.1 \mathrm{~ms}$ in duration, $1.5 \mathrm{~mW}$ at sample plane) was directed at a dendritic spine in the presence of $2 \mathrm{mM}$ of MNI-glutamate (MNI-Glu) (figure 2(b)).

The evoked response is similar in amplitude and kinetics to miniature excitatory postsynaptic currents, mEPSCs, that have been previously reported for CA1 hippocampal pyramidal neurons [34] and to light-evoked EPSCs from laser scanning systems [35].

This result suggests that the $3 \mathrm{D}$ holographic system has the resolution and usable power to stimulate individual synaptic spines.

\subsection{Effective spatial resolution during photolysis of dendritic branches}

In experiments employing photolysis of caged compounds the diffusion of the photoreleased compound during the time period of illumination also needs to be taken into account when 
considering the effective spatial resolution. We first determined the lateral resolution by moving a single excitation spot $(6.5 \mathrm{~mW}, 0.5 \mathrm{~ms})$ orthogonally across a thin dendrite $(1 \mu \mathrm{m}$ in diameter, located $\sim 50 \mu \mathrm{m}$ below the surface of the brain slice) in the presence of $2 \mathrm{mM}$ of MNI-Glu, and recording the corresponding photolysis evoked current response (upper panel, figure 3(a)). The spot was moved by projecting holograms with increasing lateral shift and keeping the sample at a fixed position. The FWHM of the peak amplitude as a function of the lateral position was 3.14 $\mu \mathrm{m}$ (lower panel, figure 3(a)). The FWHM for the group data $(\mathrm{n}=6)$ was $3.15 \mu \mathrm{m}$, figure 3(b). Next, axial resolution was extracted by measuring current elicited while varying the axial position of the holographic spot (upper panel, figure 3(c)). This was done by projecting hologram with increasing defocus and keeping the sample at a fixed position. The FWHM measurement of the current response as a function of axial resolution was $8.7 \mu \mathrm{m}$ for an individual dendrite (lower panel, figure 3(c)) and $9.65 \mu \mathrm{m}$ in group data ( $\mathrm{n}=6)$ (figure 3(d)).

The observed broadening of FWHM can be accounted by the diffusion of the photoreleased compound. To roughly simulate this effect we calculated the 3D temporal broadening of the excitation spot (taking the experimental lateral and axial profiles) due to the diffusion of uncaged glutamate based on Fick's second law and the diffusion coefficient $\mathrm{D}$ of glutamate in brain tissue $\left(D=0.33 \mu \mathrm{m}^{2} / \mathrm{ms}[36,37]\right)$. We found that a spot of initial lateral FWHM and axial FWHM of 0.3 $\mu \mathrm{m}$ and $1.2 \mu \mathrm{m}$, respectively, in the temporal interval of $1 \mathrm{~ms}(3 \mathrm{~ms}$ ) (current rise time) reaches corresponding values of $2.1 \mu \mathrm{m}(3.4 \mu \mathrm{m})$ and $5.7 \mu \mathrm{m}(9.7 \mu \mathrm{m})$. These values are in agreement with experimental findings, considering the $\sim 1 \mu \mathrm{m}$ diameter of the target dendrite.

\subsection{Simultaneous $3 D$ stimulation}

In order to demonstrate the 3D capability of digital holography, we carried out focal photolysis directed at multiple sites located on different axial planes simultaneously. First, we directed photolysis on two spines on a single dendritic branch but which were located in two different axial planes separated by $15 \mu \mathrm{m}$ (figure 4(a)). The two dendritic spines were first stimulated individually and then simultaneously $(3.2 \mathrm{~mW}$ per spot, $0.3 \mathrm{~ms})$ in the presence of $2 \mathrm{mM}$ of MNIGlu. We predicted that if instantaneous 3D photolysis was effective, then the arithmetic sum of the individual responses would equal that of the response to simultaneous photolysis at subthreshold amplitudes [9]. Indeed, the spatially summed response of the two axially displaced spines was the same as the arithmetic sum of the two individual responses (figure 4(a) and 4(b)). 
We also predicted that attempts to simultaneously stimulate the two spines using photolysis within a single plane would result in a response that corresponds to that for a single spine. Indeed, photolysis directed $15 \mu \mathrm{m}$ above the second spine resulted in no observable additional contribution from the second spine (figure 4(a) and (b)).

We then examined the effectiveness of $3 \mathrm{D}$ photolysis using multiple spots on two dendritic branches located in different axial planes $>20 \mu \mathrm{m}$ apart. The stimulus strength for the two branches was greater than that employed for dendritic spine stimulation. Photolysis was directed on 2 locations on the shaft of each dendrite (1.6 $\mathrm{mW}$ per spot). The response to simultaneous photolysis of the two branches was slightly lower than the arithmetic sum of the responses to stimulating the two branches independently (figure 4(c), individual neuron, figure 4(d), group data). These data again demonstrate the effectiveness of the axial resolution of the 3D stimulation. Photolysis with all the spot held in the same plane $(\mathrm{z}=0)$ resulted in no observable additional response beyond what was expected from the target in that plane. The small degree of sub-linear summation (10-15\%) between branches but not between spines is not a technical issue of photolysis, but a phenomenon of dendritic excitability (to be discussed in greater detail in figure $\mathbf{5}$ ).

\section{Multibranch dendritic integration}

One fundamental issue in neuroscience is how inputs arriving on different dendritic branches are summed. However, it has not been possible to properly address the latter issue using multisite photolysis because currently photolysis techniques are limited to 2D light patterns and a situation where significant portions of multiple dendrites is positioned in a single plane is unlikely. The only study of integration between two dendritic branches was carried out using two separate iontophoretic electrodes [38]. However this approach does not provide the full range of control with respect to the location, timing, and strength of stimulation.

Here we demonstrate that 3D holographic technology overcomes this fundamental limitation allowing us to conduct integration of multiple dendritic branches.

Figure 5(a) shows a representative set of recording of five different apical oblique dendrites from acute hippocampal slices. The locations of focal photostimulation (two spots for each branch, pulse duration $0.3-1 \mathrm{~ms}$; total power, $6.5 \mathrm{~mW}$ ) on the five dendrites are schematically illustrated on the right. The electrical responses to stimulation of the individual branches are 
shown in the lower traces to the left. And the responses to the simultaneous stimulation of multiple branches (i.e. spatial integration) are shown in the upper left traces.

It is surprising, on first inspection, that multi-branch summation of the oblique dendrites is sublinear whereas summation on the same branch typically is linear to supralinear [9] (Figure 5(a)). Passive cable theory predicts that for inputs separated by wider distances should sum more linearly [39]. The observation that multi-branch integration can be significantly sub-linear suggests that some active mechanisms must be triggered by sub-threshold inputs. A similar sublinear summation, albeit less prominent, was observed using iontophretic electrodes to stimulate two branches [38]. It was suggested that the mechanism for the sub-linear summation in the latter study was due to the recruitment of the transient $\mathrm{I}_{\mathrm{A}}$ type potassium channels highly expressed in the apical dendrites of hippocampal pyramidal neurons [40]. The more robust sub-linear summation due to summation between larger number of branches could also be eliminated with the addition of 4-aminopyridine, an antagonist of the $\mathrm{I}_{\mathrm{A}}$ potassium conductance (unpublished data).

\section{$\underline{\text { Summary }}$}

In this study we presented an experimental system for the generation of 3D holographic photostimulation patterns. We demonstrated the flexibility of this approach in different experimental configurations ranging from the excitation of a single dendritic spine to the simultaneous stimulation of multiple dendritic branches of hippocampal neurons.

We demonstrated that the use of 3D stimulation to control neuronal excitability permits one to study spatial integration on a single dendritic branch without the need to keep all of the sites of photostimulation in the same optical plane. We also demonstrated that $3 \mathrm{D}$ holographic light patterns permit one to investigate how inputs arriving on different dendritic branches are summed. This is a fundamental question in neuroscience and to date has been inaccessible with traditional optical methods due to the unlike possibility of multiple interconnected dendrites forming in a single optical plane.

Other examples for future applications include the study of functional circuitry to map connectivity of neurons within a 3D space rather than only those within a 2D plane.

The experiments described in this paper have been performed in single photon excitation and thus where limited to the first $40 \mu \mathrm{m}$ of the brain slices. It is believed that the use of $3 \mathrm{D}$ 
holography in two photon excitation as proposed by V. Daria et al. [26] will permit to easily overcome this limit.

3D light patterning can also be achieved with an AODs based set up [41, 42], however, besides the already mentioned limits in temporal resolution, this approach requires the set up of a bulky system of difficult alignment. It has significant power losses due to the numerous optical components. In the case of two photon excitation, requires compensation for chromatic aberrations and pulse dispersion which are negligible when phase modulation is achieved through the use of a SLM [43]. Finally the use of a SLM also allows for simultaneous control of light patterning and correction for optical aberrations, as already suggested for adaptive correction of optical trap efficiency [44].

\section{Figure legends}

Figure 1: 3D holographic light patterns.

(a) Schematic representation explaining how the coordinates at the pupil plane are related to the spherical wave front in the sample plane.

(b) 3D distribution of spots generated in a cubic pattern. 3D visualization of spots is obtained by using a double microscope set up where the upper objective generating the holographic light distribution is scanned (at step of $500 \mathrm{~nm}$ ) through a fluorescent layer positioned at the focal plane of the collecting objective. The recorded stack is projected from two perspectives to better illustrate the point spread function of the individual spots. Upper panel: side view, lower panel: axial view. 3D image reconstruction has been made with BioImageXD Project, version 0.9.0 beta. Corners of the cube are separated by $20 \mu \mathrm{m}$.

Lateral and axial FWHM where comprised between $0.3 \mu \mathrm{m}$ to $\sim 0.4 \mu \mathrm{m}$ and $1.2 \mu \mathrm{m}$ to $\sim 2 \mu \mathrm{m}$, respectively.

(c) Measured lateral diffraction efficiency, $\delta=\mathrm{I}_{\text {spot }} / \mathrm{I}_{0}$, as a function of the lateral position, dx, of a spot generated at coordinates $\mathrm{y}=5 \mu \mathrm{m}$ and $\mathrm{z}=0$ and theoretical curve (red line) taking into account the axial diffraction efficiency of the SLM (equation (3)). (d) Measured axial diffraction efficiency as a function of the axial position, $\mathrm{dz}$, of a spot generated at coordinates $\mathrm{x}=0$; $\mathrm{y}=$ 
$5 \mu \mathrm{m}$ and theoretical curve (green line) taking into account the axial diffraction efficiency of the SLM.

Figure 2: Targeting individual dendritic spines.

(a) Left panel: wide field fluorescence image of a dendritic segment loaded with Cascade Blue hydrazide via the patch electrode. Middle panel: fluorescent image of the laser spot illuminating the dendritic spine. Right panel: overlay of left and mid panel. (d) Response to stimulation of a single dendritic spine to a $1.5 \mathrm{~mW}, 0.1 \mathrm{~ms}$ laser pulse in presence of $2 \mathrm{mM}$ MNI-glutamate.

Figure 3: Effective spatial resolution during photolysis.

(a) Upper panel: current responses to a constant stimulus $(6.5 \mathrm{~mW}, 0.5 \mathrm{~ms})$ as the positions of the stimulation are moved orthogonally across a dendritic branch. Lower panel: Peak current plotted as a function of lateral position for a single dendrite. (b) Group data as a function of lateral position. (c) Upper panel: current responses obtained by axially moving the position of the stimulation through a dendrite. Lower panel: current response for a single dendrite. (d): group data as a function of axial position.

Figure 4: Simultaneous photostimulation at two different planes.

(a) Two dendritic spines on a steeply inclined dendrite are stimulated separately and then simultaneously. Spine \#2 is located $15 \mu \mathrm{m}$ below spine \#1, located at the objective focal plane $(0$ $\mu \mathrm{m})$. Simultaneous $3 \mathrm{D}$ photolysis of these two spines $(3.2 \mathrm{~mW}$ per spot, $0.3 \mathrm{~ms})$ produced linear summation which equaled the expected arithmetic sum of the individual response to the two spots. (b) Group data, $n=3$. Summation across two planes at an average of $19 \mu \mathrm{m}$ axial separation is linear, whereas stimulation held at a single axial position $(0 \mu \mathrm{m})$ does not add any observable increased response. (c) The summation of the responses recorded by exciting with two spots (3.2 $\mathrm{mW}$ ) on two dendrites separated by $20 \mu \mathrm{m}$ in the axial direction is slightly sublinear to the expected response. (d) Group data, $n=4$.

Figure 5: Spatial summation on multiple dendritic branches oriented in different directions and focal planes. 
(a) Voltage responses of a single CA1 pyramidal neuron to stimulation of individual branches (lower left) and when multiple branches are stimulated simultaneously (0.3-1ms; total power, $6.5 \mathrm{~mW}$ ) (upper left). The arithmetic sum of the individual branches compared to the actual response to simultaneous stimulation of multiple branches of this cell is plotted as the red circle in (b). (b) Group data from seven cells. Sublinear summation is consistently observed up to an expected depolarization of $30 \mathrm{mV}$.

\section{Acknowledgements}

We thank Roberto Di Leonardo for helpful discussions on the calculation algorithm and Francesca Anselmi and Aurélien Bègue for the help in setting up the electrophysiological system

for the experiments realized in Paris. EP was supported by a fellowship from the Fondation pour la Recherche Médicale. CMT acknowledges the financial support from VA Merit Review and NIH, VE and CMT acknowledge the financial support from Paris school of neuroscience and Consulate General of France in Los Angeles.

\section{References}

1. M. Scanziani and M. Hausser, "Electrophysiology in the age of light," Nature 461, 930939 (2009).

2. S. Gasparini and J. C. Magee, "State-dependent dendritic computation in hippocampal CA1 pyramidal neurons," J Neurosci 26, 2088-2100 (2006).

3. T. Branco, B. A. Clark, and M. Hausser, "Dendritic discrimination of temporal input sequences in cortical neurons," Science 329, 1671-1675.

4. S. Shoham, D. H. O'Connor, D. V. Sarkisov, and S. S. Wang, "Rapid neurotransmitter uncaging in spatially defined patterns," Nat Methods 2, 837-843 (2005).

5. B. E. Losavio, V. Iyer, S. Patel, and P. Saggau, "Acousto-optic laser scanning for multisite photo-stimulation of single neurons in vitro," J Neural Eng 7, 045002 (2010).

6. M. Matsuzaki, G. C. Ellis-Davies, and H. Kasai, "Three-dimensional mapping of unitary synaptic connections by two-photon macro photolysis of caged glutamate," J Neurophysiol 99, 1535-1544 (2008).

7. V. Nikolenko, K. E. Poskanzer, and R. Yuste, "Two-photon photostimulation and imaging of neural circuits," Nat Methods 4, 943-950 (2007).

8. F. F. Trigo, J. E. Corrie, and D. Ogden, "Laser photolysis of caged compounds at 405 $\mathrm{nm}$ : photochemical advantages, localisation, phototoxicity and methods for calibration," J Neurosci Methods 180, 9-21 (2009).

9. A. Losonczy and J. C. Magee, "Integrative properties of radial oblique dendrites in hippocampal CA1 pyramidal neurons," Neuron 50, 291-307 (2006). 
10. P. J. Sjostrom, E. A. Rancz, A. Roth, and M. Hausser, "Dendritic excitability and synaptic plasticity," Physiol Rev 88, 769-840 (2008).

11. N. Grossman, V. Poher, M. S. Grubb, G. T. Kennedy, K. Nikolic, B. McGovern, R. B. Palmini, Z. Gong, E. M. Drakakis, M. A. Neil, M. D. Dawson, J. Burrone, and P. Degenaar, "Multi-site optical excitation using ChR2 and micro-LED array," J Neural Eng 7, 16004 (2010).

12. L. J. Horneck, "128 X 128 Deformable Mirror Device," IEEE Trans. Electron Devices ED-30, 539 (1983).

13. N. Farah, I. Reutsky, and S. Shoham, "Patterned optical activation of retinal ganglion cells," Conf Proc IEEE Eng Med Biol Soc 2007, 6369-6371 (2007).

14. S. Wang, S. Szobota, Y. Wang, M. Volgraf, Z. Liu, C. Sun, D. Trauner, E. Y. Isacoff, and $\mathrm{X}$. Zhang, "All optical interface for parallel, remote, and spatiotemporal control of neuronal activity," Nano Lett 7, 3859-3863 (2007).

15. Z. V. Guo, A. C. Hart, and S. Ramanathan, "Optical interrogation of neural circuits in Caenorhabditis elegans," Nat Methods 6, 891-896 (2009).

16. C. W. Liang, M. Mohammadi, M. D. Santos, and C.-M. Tang, "Patterned photostimulation with digital micromirror devices to investigate dendritic integration across branch points," J of Visualized Experiments in press(2011).

17. J. E. Curtis, B. A. Koss, and D. G. Grier, "Dynamic holographic optical tweezers.," Opt. Communi. 207, 169 (2002).

18. H. Melville, G. F. Milne, G. C. Spalding, W. Sibbett, K. K. Dholakia, and D. D. McGloin, "Optical trapping of three dimensional structures using dynamic holograms.," Opt. Express 11, 3562 (2003).

19. J. Leach, G. Sinclair, P. Jordan, J. Courtial, M. J. M. J. Padgett, J. Cooper, and Z. J. Laczik, " 3D manipulation of particles into crystal structures using holographic optical tweezers," Opt. Express 12, 220 (2004).

20. V. Emiliani, D. Cojoc, E. Ferrari, V. Garbin, C. Durieux, M. Coppey, and E. Di Fabrizio, "Wave front engineering for living cells microscopy," Optics Express 13, 1395-1405 (2005).

21. P. J. Rodrigo, V. R. Daria, and J. Glückstad, "Four-dimensional optical manipulation of colloidal particles," Applied Physics Letters 86, 074103 (2005).

22. E. Papagiakoumou, F. Anselmi, A. Begue, V. de Sars, J. Gluckstad, E. Y. Isacoff, and V. Emiliani, "Scanless two-photon excitation of channelrhodopsin-2," Nat Methods 7, 848854 (2010).

23. W. Gobel, B. M. Kampa, and F. Helmchen, "Imaging cellular network dynamics in three dimensions using fast 3D laser scanning," Nat Methods 4, 73-79 (2007).

24. C. Lutz, T. S. Otis, V. de Sars, S. Charpak, D. A. DiGregorio, and V. Emiliani, "Holographic photolysis of caged neurotransmitters," Nat Methods 5, 821-827 (2008).

25. L. Golan, I. Reutsky, N. Farah, and S. Shoham, "Design and characteristics of holographic neural photo-stimulation systems," J Neural Eng 6, 66004 (2009).

26. V. R. Daria, C. Stricker, R. Bowman, S. Redman, and H. A. Bachor, "Arbitrary multisite two-photon excitation in four dimensions," Applied Physics Letters 95, - (2009).

27. V. Nikolenko, B. O. Watson, R. Araya, A. Woodruff, D. S. Peterka, and R. Yuste, "SLM Microscopy: Scanless Two-Photon Imaging and Photostimulation with Spatial Light Modulators," Front Neural Circuits 2, 5 (2008).

28. M. Zahid, M. Velez-Fort, E. Papagiakoumou, C. Ventalon, M. C. Angulo, and V. Emiliani, "Holographic photolysis for multiple cell stimulation in mouse hippocampal slices," PLoS One 5, e9431 (2010). 
29. B. Richards and E. Wolf, "Electromagnetic diffraction in optical systems. II. Structure of the image field in an aplanatic system," Proc. Roy. Soc. Lond. Ser. A 253, 358-379 (1959).

30. J. Leach, K. Wulff, G. Sinclair, P. Jordan, J. Courtial, L. Thomson, G. Gibson, K. Karunwi, J. Cooper, Z. J. Laczik, and M. J. M. J. Padgett, "Interactive approach to optical tweezers control," Appl Opt 45, 897 (2006).

31. E. J. Botcherby, R. Juskaitis, M. J. Booth, and T. Wilson, "Aberration-free optical refocusing in high numerical aperture microscopy," Optics Letters 32, 2007-2009 (2007).

32. R. Di Leonardo, F. Ianni, and G. Ruocco, "Computer generation of optimal holograms for optical trap arrays," Optics Express 15, 1913-1922 (2007).

33. M. Born and E. Wolf, Principles of optics : electromagnetic theory of propagation, interference and diffraction of light, 7th ed. ed. (Cambridge University Press, Cambridge, 1999), p. 1200p.

34. R. C. Carroll, R. A. Nicoll, and R. C. Malenka, "Effect of PKA and PKC on miniature excitatory postsynaptic currents in CA1 pyramidal cells," J Neurophysiol 80, 2797 (1998).

35. A. A. Bagal, J. P. Kao, C. M. Tang, and S. M. Thompson, "Long-term potentiation of exogenous glutamate responses at single dendritic spines," Proc Natl Acad Sci U S A 102, 14434-14439 (2005).

36. T. S. Otis, Y. C. Wu, and L. O. Trussell, "Delayed clearance of transmitter and the role of glutamate transporters at synapses with multiple release sites," J Neurosci 16, 1634-1644. (1996).

37. D. A. DiGregorio, J. S. Rothman, T. A. Nielsen, and R. A. Silver, "Desensitization properties of AMPA receptors at the cerebellar mossy fiber granule cell synapse.," J. Neurosci. 27, 8344-8357 (2007).

38. S. Cash and R. Yuste, "Input summation by cultured pyramidal neurons is linear and position-independent," J Neurosci 18, 10-15 (1998).

39. W. Rall, "Distinguishing theoretical synaptic potentials computed for different somadendritic distributions of synaptic input," J Neurophysiol 30, 1138-1168 (1967).

40. D. A. Hoffman, J. C. Magee, C. M. Colbert, and D. Johnston, "K+ channel regulation of signal propagation in dendrites of hippocampal pyramidal neurons," Nature 387, 869-875 (1997).

41. G. D. Reddy and P. Saggau, "Fast three-dimensional laser scanning scheme using acousto-optic deflectors," J Biomed Opt 10, 064038 (2005).

42. P. A. Kirkby, K. M. Srinivas Nadella, and R. A. Silver, "A compact Acousto-Optic Lens for 2D and 3D femtosecond based 2-photon microscopy," Opt Express 18, 13721-13745 (2010).

43. E. Papagiakoumou, V. de Sars, D. Oron, and V. Emiliani, "Patterned two-photon illumination by spatiotemporal shaping of ultrashort pulses," Optics Express 16, 2203922047 (2008).

44. K. D. Wulff, D. G. Cole, L. C. Robert, R. DiLeonardo, L. Jonathan, J. Cooper, G. Graham, and M. J. Padgett, " Aberration correction in holographic optical tweezers," Opt. Express 14, 4170 (2006). 
(a)

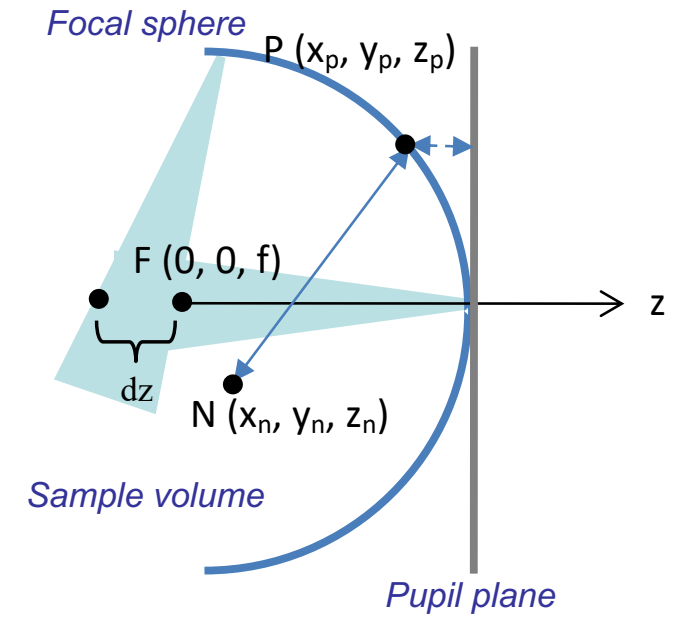

(c)

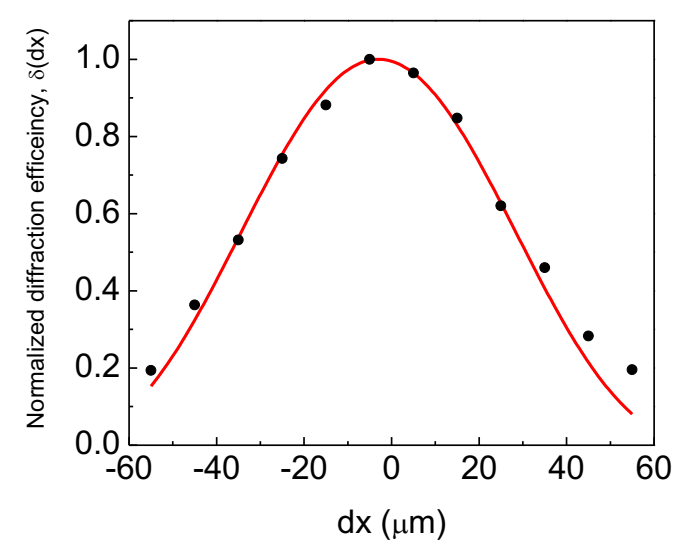

(b)

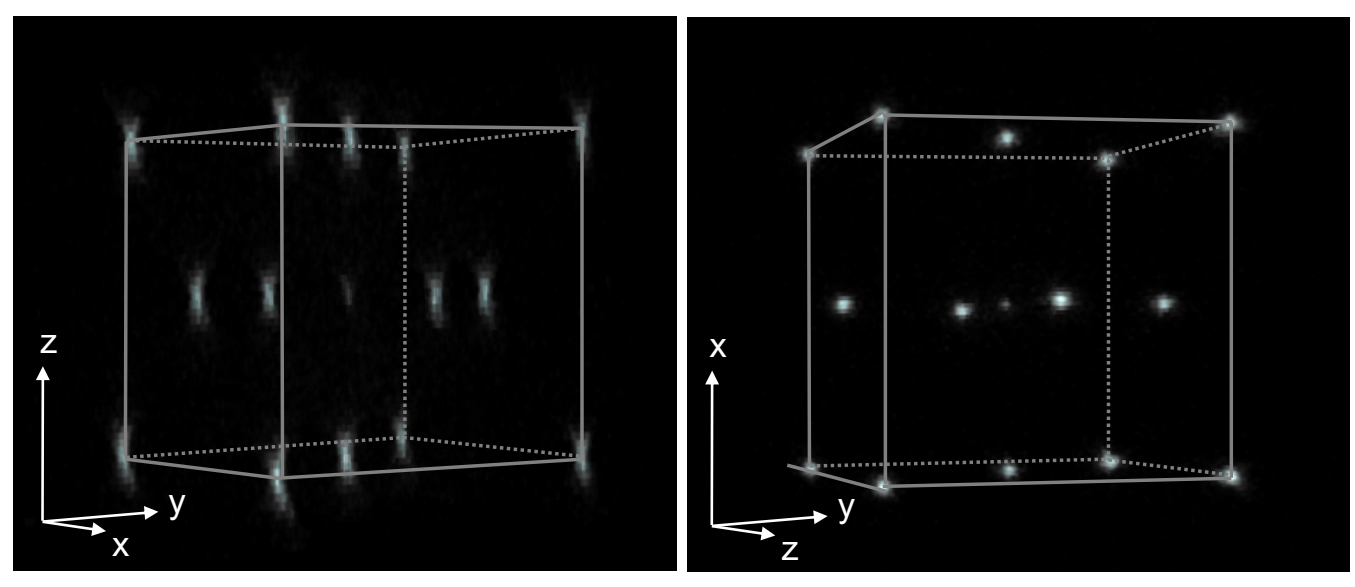

(d)

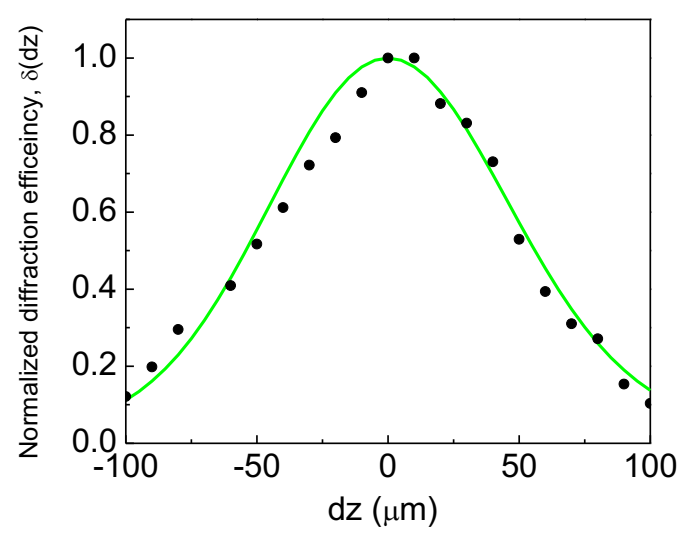

Figure 1 
(a)

$$
\begin{array}{cc}
\text { Cascade blue- } & \text { Holographic } \\
\text { labeled dendrite } & \text { excitation spot }
\end{array}
$$

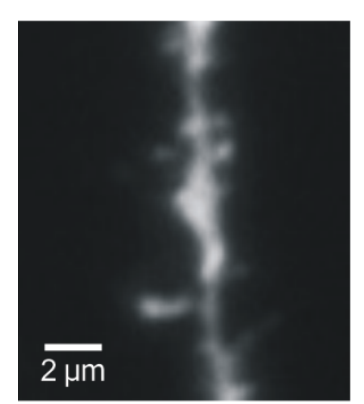

(b)

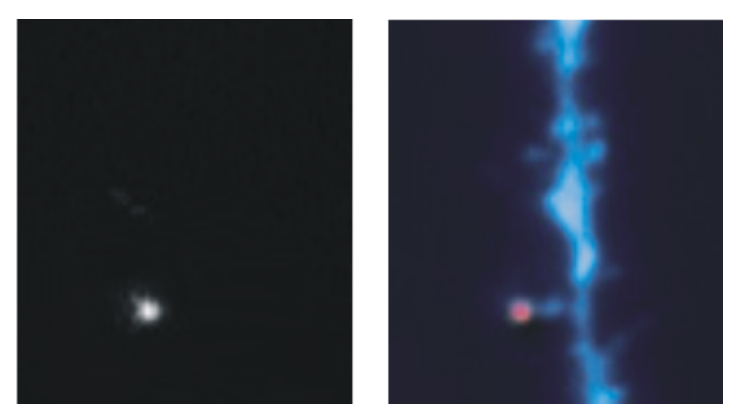

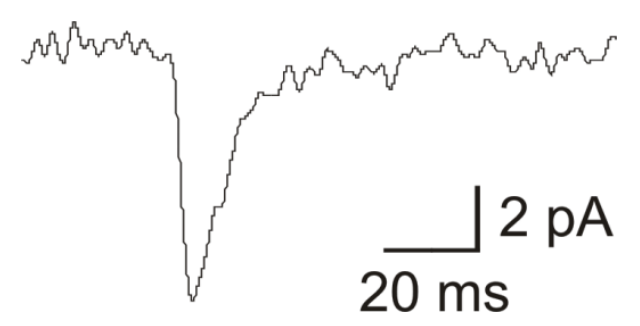

Figure 2 
(a)
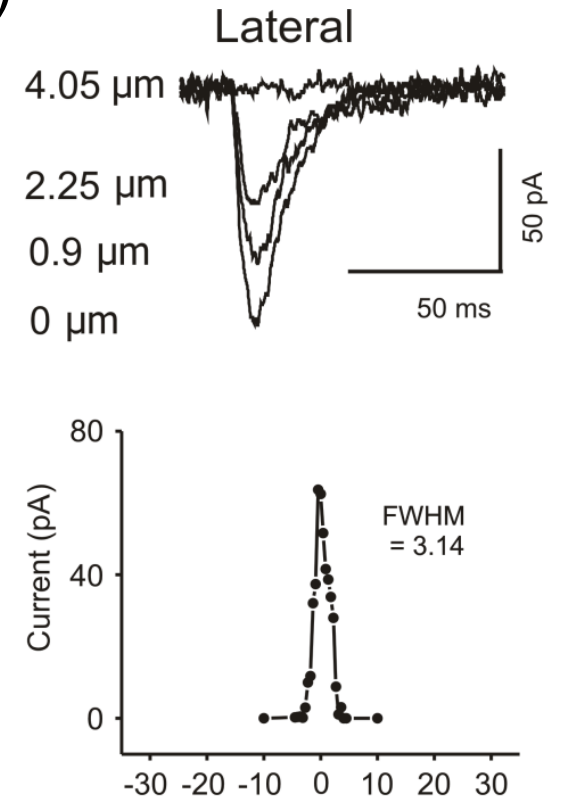

(b)

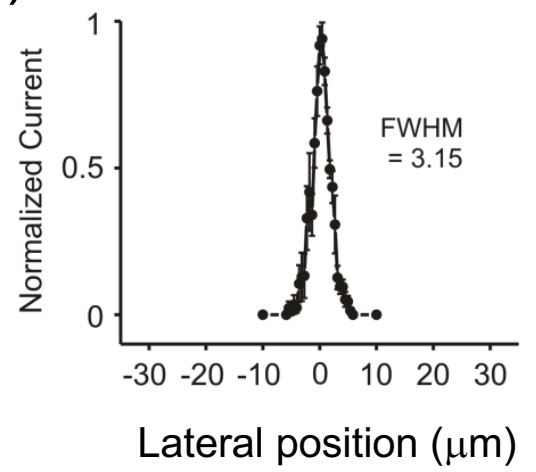

(c)
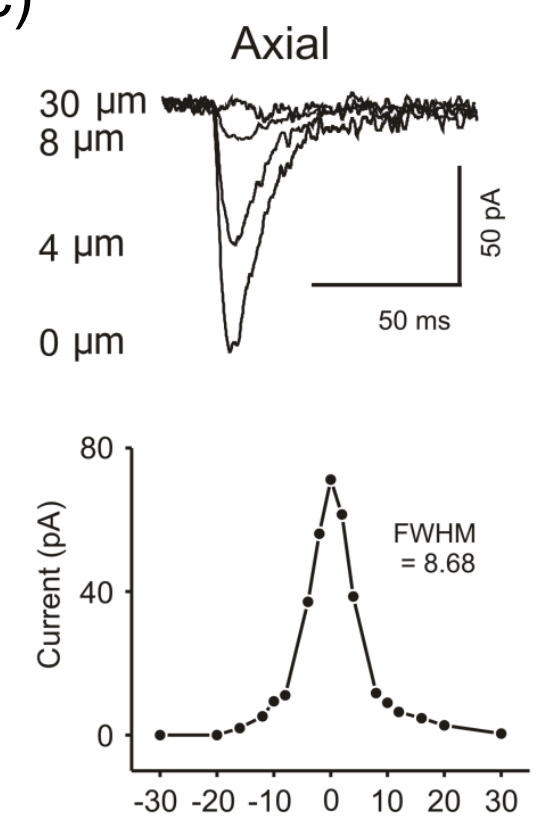

(d)

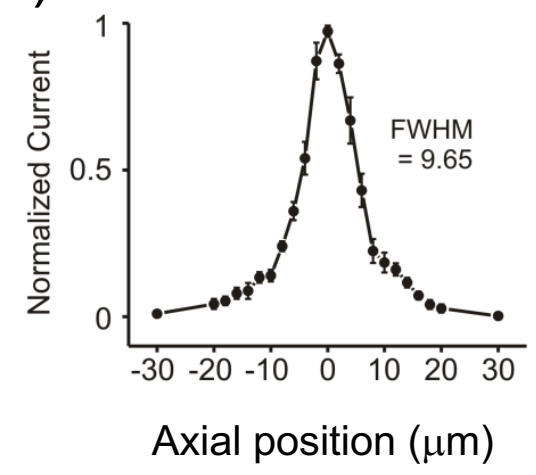

Figure 3 
(a)
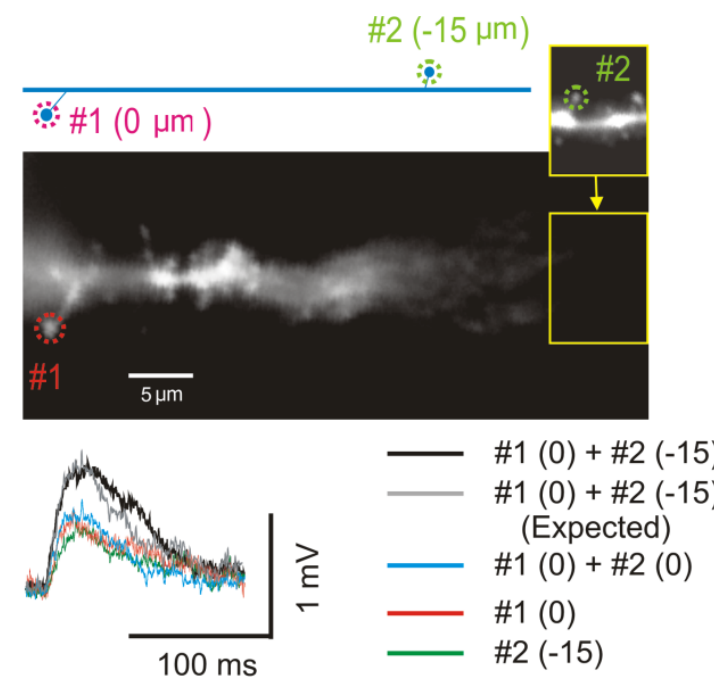

(b)

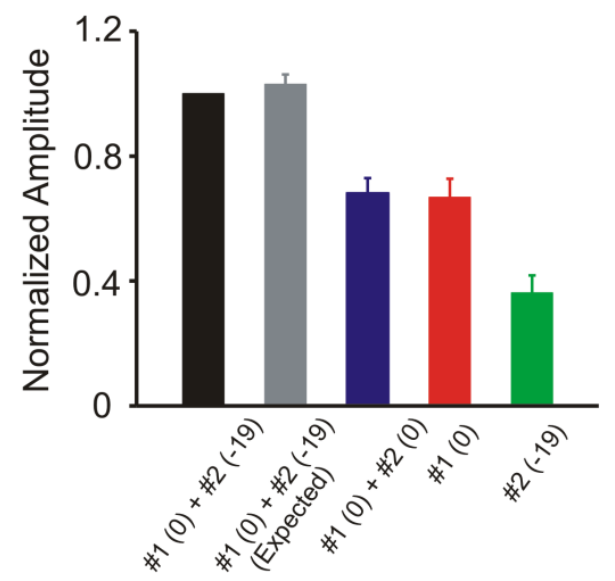

(c)

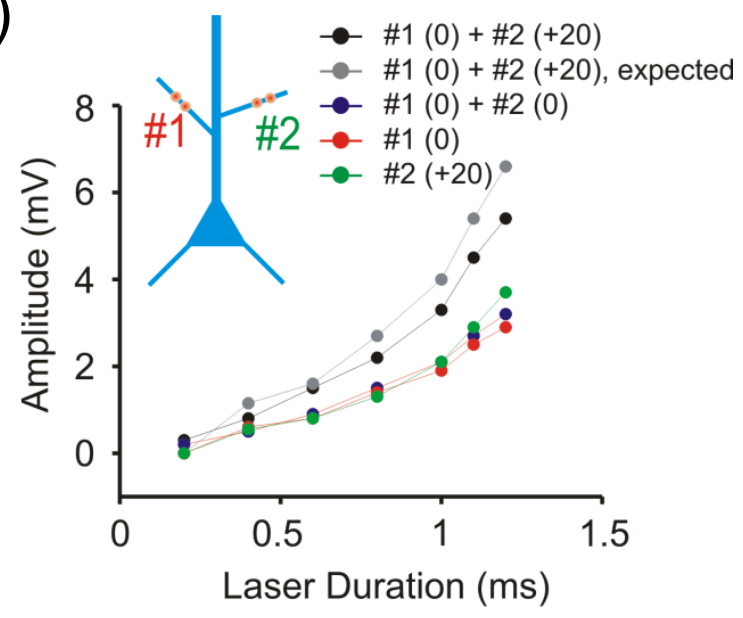

(d)

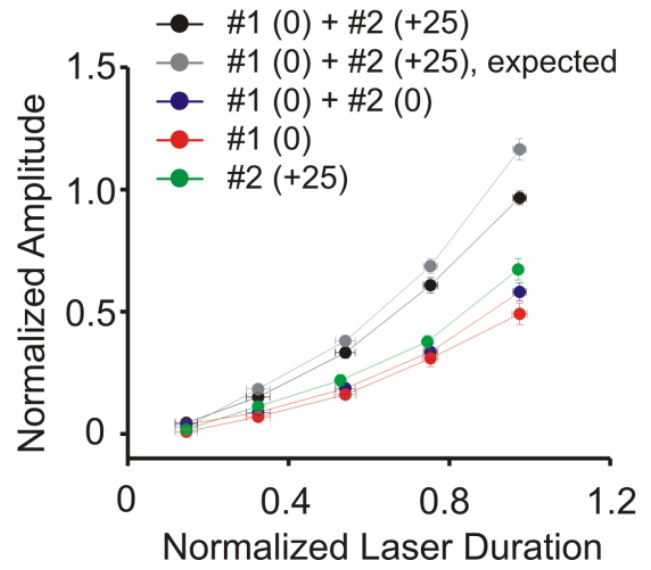

Figure 4 
(a)

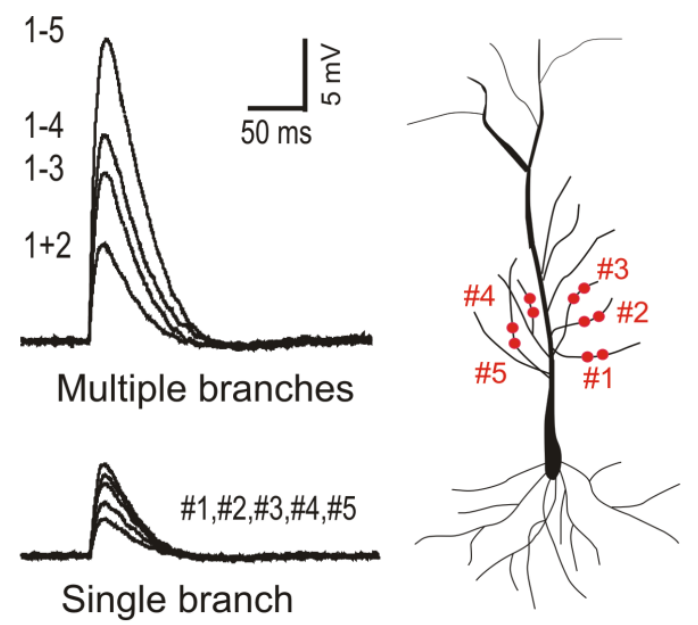

(b)

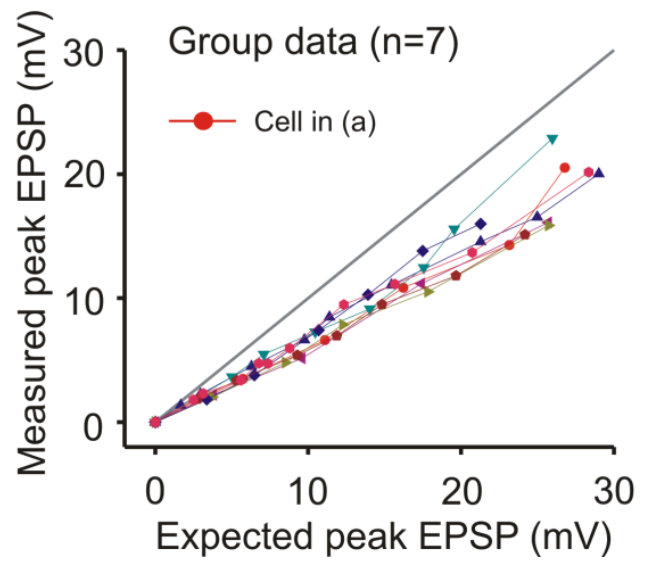

Figure 5 